\title{
On Innervation of Taste-Buds in Larynx in Dog
}

\author{
By \\ Hideo Koizumi \\ (小泉 秀婔) \\ From the Anatomical Laboratory of Prof. H. Seto, \\ University, Sendai \\ (Received for publication, December 29, 1952)
}

Since Verson (1868) discovered the existence of taste-bud-like organs on the laryngeal face of epiglottis, many subsequent studies confirmed the existence. However, opinion was divided on their physiological meaning, that is, on the problem whether they were true taste sense organs as the taste-buds on the dorsum linguae. For example, Simanowsky') examined the nerves for the so-called goblet organs in larynx in man and mammals histologically, and came to the supposition that they were related with pressure or tactile sense rather than the sense of taste. Michelson (1891 $\left.{ }^{21}\right)$, however, made it clear that taste sense organs exist also on the laryngeal side of epiglottis from the results of his experiment on human larynx. This fact is beyond doubt from the histological structure of the organs.

Recently, Sasaki ${ }^{3)}$ of this laboratory conducted a very minute histological research on the innervation of the taste-buds found in larynx of human adults and arrived at many new observations. I made a similar study, with stress upon the sensory innervation, on canine larynx, in succession to the study on the nerve supply of canine larynx, ${ }^{4)}$ utlizing the same preparations stained with Seto's silver method, as in my previous study. I will hereunder describe my views in comparison with those obtained by Sasaki in his study on human larynx.

As to the distribution of taste-buds in canine larynx, they were found most abundant on the laryngeal side of epiglottis, especially in its upper parts, next in plica ventricularis and on the innerside of cartilago arytaenoides, and in a number also in the glottid part and pars intercartilaginea rimae glottidis lined by the stratified flat epithelium. The above is in conformance with the description on the subject by Davis ${ }^{5}$, and is not much different from what has been reported on the human larynx (Sasaki $\left.^{3)}\right)$.

The development of the nerve elements to such taste-buds is lower in dog than in man. They take their origin in the subepithelial pleuxs 
and penetrate close to the base of the taste-buds, some ending in simple glomerular terminations or plexus-like terminations, but in most cases ending in unbranched or simple branched terminations. In rare cases, however, they penetrate further into the taste-buds or the epithelium around them, to end as intra- and extragemmal fibres.

For example, Fig. 1 shows the terminal state of taste nerve fibres to a taste-bud found in plica ventricularis. One or two thick sensory fibres coming from the subepithelial plexus reach the papilla under the taste-bud, showing peculiar change in size and running a winding course, and they finally pass into a simple glomerular termination consisting of thick fibres and thin fibres, a part of which further penetrating into the basis of the taste-bud, to terminate as intragemmal fibres.

Fig. 2 show's the innervation of taste-buds found on the inside of cartilago arytaenoides. Thick fibres and thin fibres terminate in the tunica propria directly under the taste-buds, showing comparatively complex plexus, from which appear intra- and extragemmal fibres ending sharply or bluntly. Figs. 3 and 4 show the sensory terminations for taste-buds found on the laryngeal side of epiglottis and in pars intercartilaginea rimae glottidis respectively. The nerve elements are from a very limited number of thick sensory fibres, which end either just beneath or in the taste-buds as intragemmal fibres, sharply or bluntly, without showing any noteworthy glomerular or plexus-like formation under the taste-buds. There is seen in Fig. 5 a simple plexus-like termination composed of fine fibres, found directly under a taste-bud in the glottid part. A few intragemmal fibres are observable going out from this termination.

As clarified in the above described figures, the nerve fibres supplying the taste-buds are classifiable into thick fibres and fine fibres, as the case is also with human larynx (Sasaki $\left.{ }^{3}\right)$. In canine larynx, however, unlike the human couterpart, the former predominates vastly, and fibres belonging to the finer type are very few. Indisagreement with the opinion of Sasaki ${ }^{3}$, I presume the fine fibres also to be of sensory nature, and not vegetative fibres, because, especially as shown in Fig. 1, the fine fibres are derived from the thick fibres by branching, in the most part.

The taste-buds in canine larynx may be classified into the four types as follows, according to the mode of sensory innervation: 1. The tastebuds best provided with sensory nerves, being supplied by both the thick and fine fibres (Figs. 1 and 2); 2. These provided with thick fibres only (Figs. 3 and 4); 3. Those provided with fine fibres only (Fig. 5) and 4. Those not supplied at all, that is, the most poorly innervated type.

This classification is in harmony with that by Sasaki of human laryngeal taste-buds. However, by human larynx, the laryngeal side of epiglottis is provided with Types I and II of the above taste-buds, and is 
Fig. 1

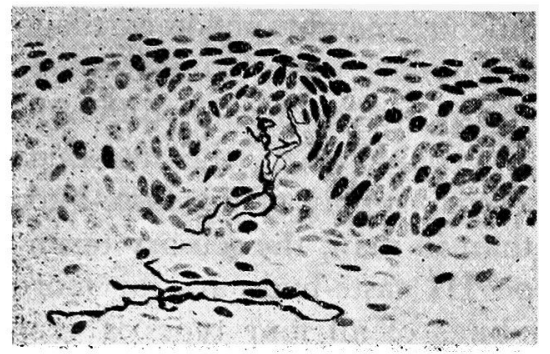

Fig. 2

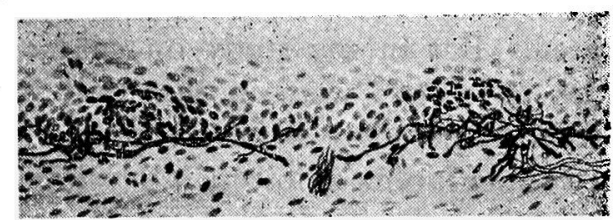

Fig. 1. A simple glomerular termination to a taste-bud found in plica ventricularis in dog. Seto's impregnation. X 500, reduced to $2 / 3$.

Fig. 2. Plexus-like terminations composed of thick fibres formed under two tastebuds on the inside of cartilago arytaenoides in dog. Intra- as well as extragemmal fibres are discernible. Same staining. $\mathrm{X} 320$, reduced to $\frac{1}{2}$.

Fig. 3

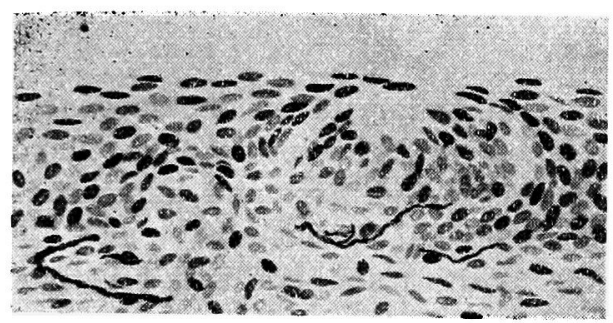

Fig. 4

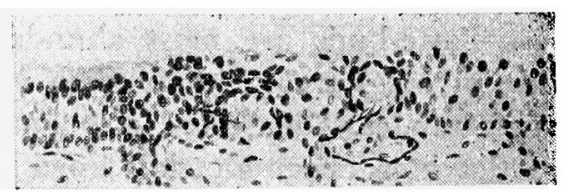

Fig. 3. Simple branched termination composed of thick fibres formed under a tastebud found on the laryngeal side of epiglottis in dog. Same staining. X 500, reduced to $\frac{1}{2}$.

Fig. 4. Unbranched and simple branched terminations of thick fibres formed under two taste-buds existing only in the stratified flat epithelium of pars intercartilaginea rimae glottidis in dog. Epithelia of both sides consist of multi-lined cilieted epithelium. Same staining. $\mathrm{X} 320$, reduced to $\frac{1}{2}$.

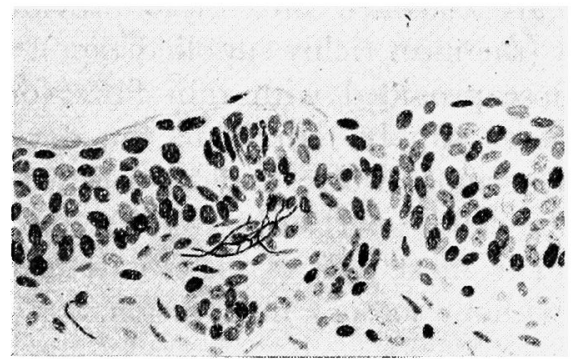

Fig. 5. Plexus-like termination composed of fine fibres formed under a taste-bud in glottid part in dog. Same staining. X 500 , reduced to $\frac{1}{2}$. 
richly supplied by sensory fibres, while the taste-buds on the glottid parts are of Types II and IV, those in the mucous membrane of cartilago arytaenoides are of Type III, that is, these parts are none too well innervated. But in my study on canine larynx, it was somewhat different. The taste-buds were in the most part of Type IV, i.e., the tate-buds lacked almost any sensory supply. The sensory supply of the taste-buds in canine larynx is lower in development than in man, the sensory terminations to the Type I, Type II and Type III taste-buds are far more simple, and the arrangement of the taste-buds of all four types is very disorderly. In man, according to Sasaki, the taste-buds are more imperfectly or degeneratively innervated as we go from the cranial to the caudal part, but in dog, this is not necessarily the case, some sensory terminations found in plica ventricularis (Fig. 1) and the inside of cartilago arytaenoides (Fig. 2) being in more complex formation than those in the laryngeal side of epiglottis. Even in the glottid part some considerably complex sensory terminations can be found.

\section{Summary}

Taste-buds are most abundant in canine larynx on the laryngeal side of epiglottis, next in plica ventricularis and the inside of cartilago arytaenoides. Some are also found in the glottid part and pars intercartilaginea rimae glottidis lined by stratified flat epithelium.

The development of sensory nerves to the taste-buds is lower than in man. The sensory fibres originate in the subepithelial plexus, and most of them pass into unbranched or simple branched terminations, though some are found ending in simple glomerular or plexus-like terminations directly under the taste-buds. In rare cases, fibres are seen further penetrating into the taste-buds, to form intra- and extragemmal fibres. These sensory fibres are composed in most part of thick fibres, and a smaller number of fine fibres, the latter as well as the former being probably of sensory nature.

The taste-buds are classified into those provided with both thick fibres and thin fibres (the most richly supplied type), those provided with only thick fibres, those provided with thin fibres only, and those that are lacking in all nerve supply. In man, the development of sensory nerves to taste-buds becomes worse as we go down from the laryngeal side of epiglottis to the caudal part of larynx, but in dog, this innervation is very disorderly. The most part of taste-buds in canine larynx is represented by those without sensory innervation.

\section{References}

1) Simanowsky, Arch. mikr. Anat., 1883, 22, 698. 
2) Michelson, Virchows Arch., 1891, 123, 389.

3) Sasaki, Tohoku Igaku Zassi, 1943, 32, 569 and 595.

4) Koizumi, Tohoku J. Exp. Med. 1953, 58, 199.

5) Davis, Arch. mikr. Anat., 1877, 14, 158. 